\title{
A Prototype High-Resolution Small-Animal PET Scanner Dedicated to Mouse Brain Imaging
}

\author{
Yongfeng Yang ${ }^{1,2}$, Julien Bec ${ }^{1}$, Jian Zhou ${ }^{1}$, Mengxi Zhang ${ }^{1}$, Martin S. Judenhofer ${ }^{1}$, Xiaowei Bai ${ }^{1}, \mathrm{Kun} \mathrm{Di}^{1}$, Yibao Wu ${ }^{1}$, \\ Mercedes Rodriguez ${ }^{1,3}$, Purushottam Dokhale ${ }^{4}$, Kanai S. Shah ${ }^{4}$, Richard Farrell ${ }^{4}$, Jinyi Qi ${ }^{1}$, and Simon R. Cherry ${ }^{1}$ \\ ${ }^{1}$ Department of Biomedical Engineering, University of California-Davis, Davis, California; ${ }^{2}$ Shenzhen Institutes of Advanced \\ Technology, Chinese Academy of Sciences, Shenzhen, China; ${ }^{3}$ Instituto de Física, Universidad Nacional Autónoma de México, \\ Mexico City, Mexico; and ${ }^{4}$ Radiation Monitoring Devices Inc., Watertown, Massachusetts
}

We developed a prototype small-animal PET scanner based on depth-encoding detectors using dual-ended readout of small scintillator elements to produce high and uniform spatial resolution suitable for imaging the mouse brain. Methods: The scanner consists of 16 tapered dual-ended-readout detectors arranged in a 61 -mm-diameter ring. The axial field of view (FOV) is $7 \mathrm{~mm}$, and the transaxial FOV is $30 \mathrm{~mm}$. The scintillator arrays consist of $14 \times 14$ lutetium oxyorthosilicate elements, with a crystal size of $0.43 \times$ $0.43 \mathrm{~mm}$ at the front end and $0.80 \times 0.43 \mathrm{~mm}$ at the back end, and the crystal elements are $13 \mathrm{~mm}$ long. The arrays are read out by $8 \times 8 \mathrm{~mm}$ and $13 \times 8 \mathrm{~mm}$ position-sensitive avalanche photodiodes (PSAPDs) placed at opposite ends of the array. Standard nuclear-instrumentation-module electronics and a custom-designed multiplexer are used for signal processing. Results: The detector performance was measured, and all but the crystals at the very edge could be clearly resolved. The average intrinsic spatial resolution in the axial direction was $0.61 \mathrm{~mm}$. A depth-of-interaction resolution of $1.7 \mathrm{~mm}$ was achieved. The sensitivity of the scanner at the center of the FOV was $1.02 \%$ for a lower energy threshold of $150 \mathrm{keV}$ and $0.68 \%$ for a lower energy threshold of $250 \mathrm{keV}$. The spatial resolution within a FOV that can accommodate the entire mouse brain was approximately $0.6 \mathrm{~mm}$ using a 3-dimensional maximum-likelihood expectation maximization reconstruction. Images of a hot-rod microphantom showed that rods with a diameter of as low as $0.5 \mathrm{~mm}$ could be resolved. The first in vivo studies were performed using ${ }^{18} \mathrm{~F}-$ fluoride and confirmed that a $0.6-\mathrm{mm}$ resolution can be achieved in the mouse head in vivo. Brain imaging studies with ${ }^{18} \mathrm{~F}-\mathrm{FDG}$ were also performed. Conclusion: We developed a prototype PET scanner that can achieve a spatial resolution approaching the physical limits of a small-bore PET scanner set by positron range and detector interaction. We plan to add more detector rings to extend the axial FOV of the scanner and increase sensitivity.

Key Words: positron emission tomography; small animal PET; high resolution; mouse; brain imaging

J Nucl Med 2016; 57:1130-1135

DOI: $10.2967 /$ jnumed.115.165886

Received Aug. 22, 2015; revision accepted Feb. 25, 2016.

For correspondence or reprints contact: Yongfeng Yang, Shenzhen Institutes of Advanced Technology, Chinese Academy of Sciences, Shenzhen 518055, China.

E-mail: yf.yang@siat.ac.cn

Published online Mar. 24, 2016.

COPYRIGHT (C) 2016 by the Society of Nuclear Medicine and Molecular Imaging, Inc.
O animal PET scanners is $1-2 \mathrm{~mm}(1-4)$. Mice are the most frequently used animal model for studies of human diseases. The strengths of the mouse as an experimental model include the fact that roughly $95 \%$ of its genes have a human homolog, techniques for its genetic manipulation are mature, it reaches breeding age quickly (accelerating the development of transgenic and knockout animals), and its housing and breeding costs are relatively low. The mouse brain is about a tenth the size of the human brain $(\sim 1.5 \mathrm{~cm}$ vs. $\sim 15 \mathrm{~cm})$. A small-animal PET scanner with approximately $0.5-\mathrm{mm}$ spatial resolution is required if one wants to image a mouse with the same relative spatial resolution as is achieved in a human using a clinical whole-body PET scanner with 5-mm spatial resolution (5,6). Because of fundamental limits stemming from the physics of the signal generation mechanism (7), it is almost impossible to achieve the same relative spatial resolution $(\sim 0.2 \mathrm{~mm})$ for a mouse brain as for a human brain imaged with a dedicated brain PET scanner that achieves an approximately 2-mm spatial resolution (8).

High spatial resolution is required to see the distribution of a radiotracer within small brain regions and to improve its quantification by reducing the partial-volume effect. Improving spatial resolution has been a major focus in small-animal PET instrumentation (9-13). The spatial resolution of a PET scanner is limited by crystal size, positron range, detector interaction, depth of interaction (DOI), crystal encoding, sampling nonuniformity, and image reconstruction (14). In this work, we focus on developing a prototype high-resolution small-animal PET scanner with spatial resolution approaching the physical limits of a preclinical PET scanner by minimizing the contribution from each of the above factors. We use depth-encoding detectors based on dual-ended readout of finely pixelated lutetium oxyorthosilicate (LSO) arrays with position-sensitive avalanche photodiodes (15-17). We have previously shown that similar detectors could resolve crystals as small as $0.5 \mathrm{~mm}$ and provide a DOI resolution of $2 \mathrm{~mm}(16)$. The contributions from crystal size, DOI, and crystal encoding are reduced using this detector design. Depth-encoding detectors can also reduce any resolution degradation or artifacts due to insufficient sampling since the sampling is improved using the DOI information. Using depth-encoding detectors with excellent DOI resolution, it is possible to design scanners with a smaller detectorring diameter to reduce both scanner cost and the contribution of detector interaction, as well as to increase sensitivity. We also are developing an iterative reconstruction algorithm with 
accurate system modeling to reduce the contribution from image reconstruction.

The scanner we are developing is dedicated to mouse brain imaging because this application requires high spatial resolution but has a manageable cost due to the relatively small FOV-and therefore detector volume-needed. Initially, we have developed a one-ring prototype scanner to test the detector approach and system concepts. In this paper, we describe the design of this prototype and demonstrate its spatial resolution and imaging performance.

\section{MATERIALS AND METHODS}

\section{Design of Prototype}

The prototype uses 16 tapered LSO crystal arrays cut into $14 \times 14$ elements $13 \mathrm{~mm}$ thick. The crystal size of the arrays is $0.43 \times 0.43 \mathrm{~mm}$ at the front end and $0.80 \times 0.43 \mathrm{~mm}$ at the back end. Two of the 4 long crystal faces are polished. The other 4 crystal faces are left unpolished, and Lumirror E60 film (Toray Industries Inc.) is used as an intercrystal reflector. Details on the array fabrication have been published previously $(15,16)$. The arrays are read out by position-sensitive avalanche photodiodes (PSAPDs), with active areas of $8 \times 8 \mathrm{~mm}$ and $13 \times 8 \mathrm{~mm}(18)$, placed at opposite ends of the array so that the DOI can be estimated. Figure 1 shows photographs of an array and the 2 different sizes of PSAPD. The physical dimensions of the 2 PSAPDs are $10 \times 11.5 \times 1.4 \mathrm{~mm}$ and $15.2 \times 16.7 \times 1.4 \mathrm{~mm}$.

Figure 1 also shows the detector arrangement and the completed scanner. The ring diameter is $61 \mathrm{~mm}$, and the aperture is $40 \mathrm{~mm}$. The axial FOV is $7 \mathrm{~mm}$, and the transaxial FOV is $30 \mathrm{~mm}$. The gap between adjacent arrays is $5 \mathrm{~mm}$. For scanners with such small crystal elements, the positioning of the arrays needs to be highly accurate in order to realize the full resolution potential. An aluminum ring support was designed to hold the arrays in known and precise locations. The performance of PSAPDs (e.g., signal-to-noise ratio and gain) changes with temperature (19); therefore, the scanner gantry was kept at a stable operating temperature using an Air-Jet Crystal Cooler (FTS System, Inc.). To obtain efficient and uniform cooling of all PSAPDs,

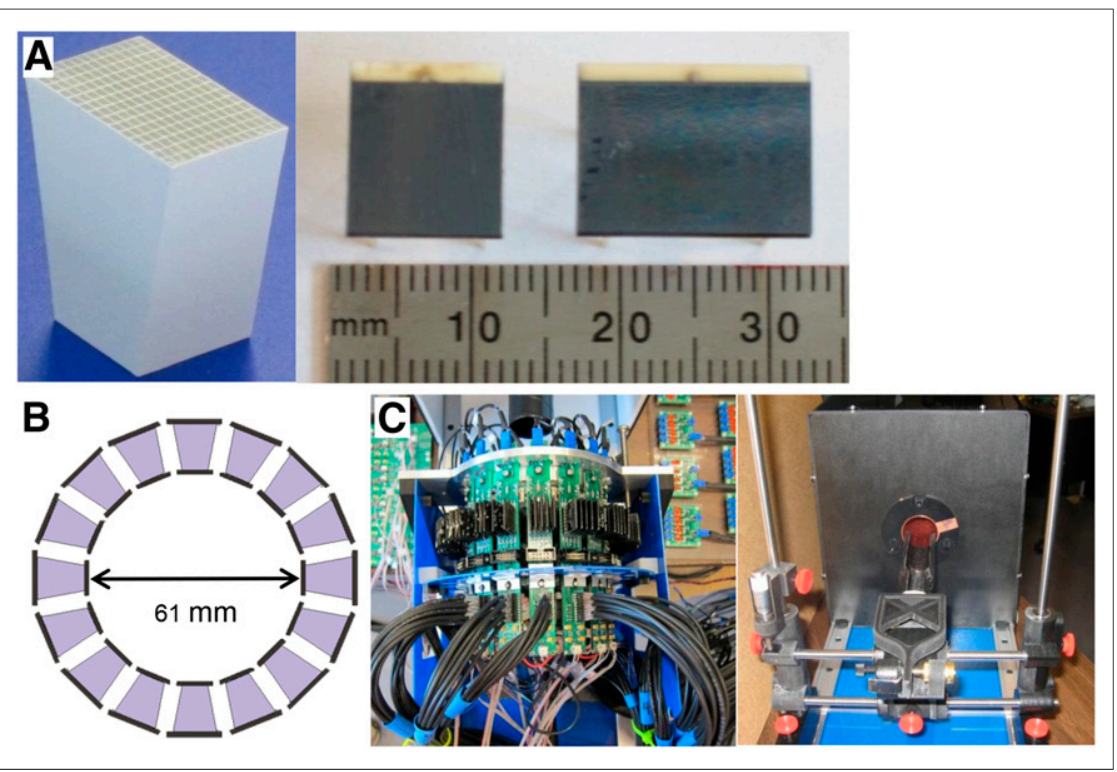

FIGURE 1. (A) Tapered LSO array and 2 different sizes of PSAPD used in prototype scanner. (B) Scale drawing of prototype one-ring scanner. (C) Photographs of scanner without cover, showing readout electronics (left), and completed scanner with animal bed (right). the cold and dry air was sent into the aluminum support structure and also was distributed to the PSAPDs at the ends of the arrays. Computed simulations of fluid dynamics were used in designing the cooling system. These simulations showed that variations in cold-air pressure across the 32 PSAPDs in the system were within $5 \%$ in the final cooling design selected. The space where all arrays, PSAPDs, and readout preamplifier boards reside also is cooled. An animal bed that can be moved in all 3 directions was also fabricated and integrated with the scanner.

The schematics of the electronics system are shown in Supplemental Figures 1 and 2. Standard nuclear-instrumentation-module electronics were used for signal processing, and a multiplexer (20) was designed and used to decrease the number of energy signals from 128 (32 PSAPDs each with 4 signal outputs) to 16 . The timing signals from the front and back PSAPDs were amplified by a CR-110 preamplifier (Cremat Inc.), summed using a linear fan-in and fan-out, and then amplified by a timing filter amplifier before being sent to a constant fraction discriminator, which produced two logic signals. One was for coincidence formation, and the other was sent to the multiplexer to determine the detector identification number. The 4 energy signals of each PSAPD were also amplified, first by the CR110 preamplifier and then by a 16-channel shaping amplifier. The 128 energy signals from the 32 PSAPDs, the 16 constant-fractiondiscriminator signals, and the coincidence-event-trigger signal were sent to the multiplexer. Whenever an event is triggered, the multiplexer determines the 2 coincidence detectors from the constant-fractiondiscriminator signals. Then, the 16 energy signals from the 2 detector modules are passed to 2 data acquisition boards, digitized, and stored as list-mode data. The multiplexer also produces 8 logic signals (the first 4 signals are for the first detector, and the last 4 signals are for the second detector), which are used to encode the detector-pair identification numbers (detector identification numbers run from 0 to 15). For example, when the detector identification number is 0 , all 4 logic signals are $0 \mathrm{~V}$, and when the detector identification number is 15 , all 4 signals are $+5 \mathrm{~V}$. The 8 logic signals were also digitized using one of the data acquisition boards and stored in the list-mode data file (21).

\section{Detector Performance}

The flood histogram, DOI resolution, and energy resolution of one detector module were measured using the same experimental setup, data acquisition system, and data analysis procedures as shown in our previous publications $(15,22)$. The experimental methods are briefly summarized below. The flood histograms and DOI responses of all 16 detectors were also measured within the scanner. For all measurements presented, the temperature was set to about $5^{\circ} \mathrm{C}$.

Flood Histograms and Energy Resolution. The measurements were performed in singles mode with the array irradiated by a $0.3-\mathrm{mm}$ diameter ${ }^{22} \mathrm{Na}$ point source placed $5 \mathrm{~cm}$ from one side of the array. To analyze the data, a preliminary flood histogram (with a low energy threshold) was first obtained from the list-mode data. From this, a crystal look-up table was generated. The list-mode data were then reprocessed using the look-up table to obtain energy spectra for each crystal in an array. The photopeak amplitude and energy resolution of all crystals were obtained by gaussian fitting of the energy spectra. In addition, the list-mode data were reanalyzed using the 


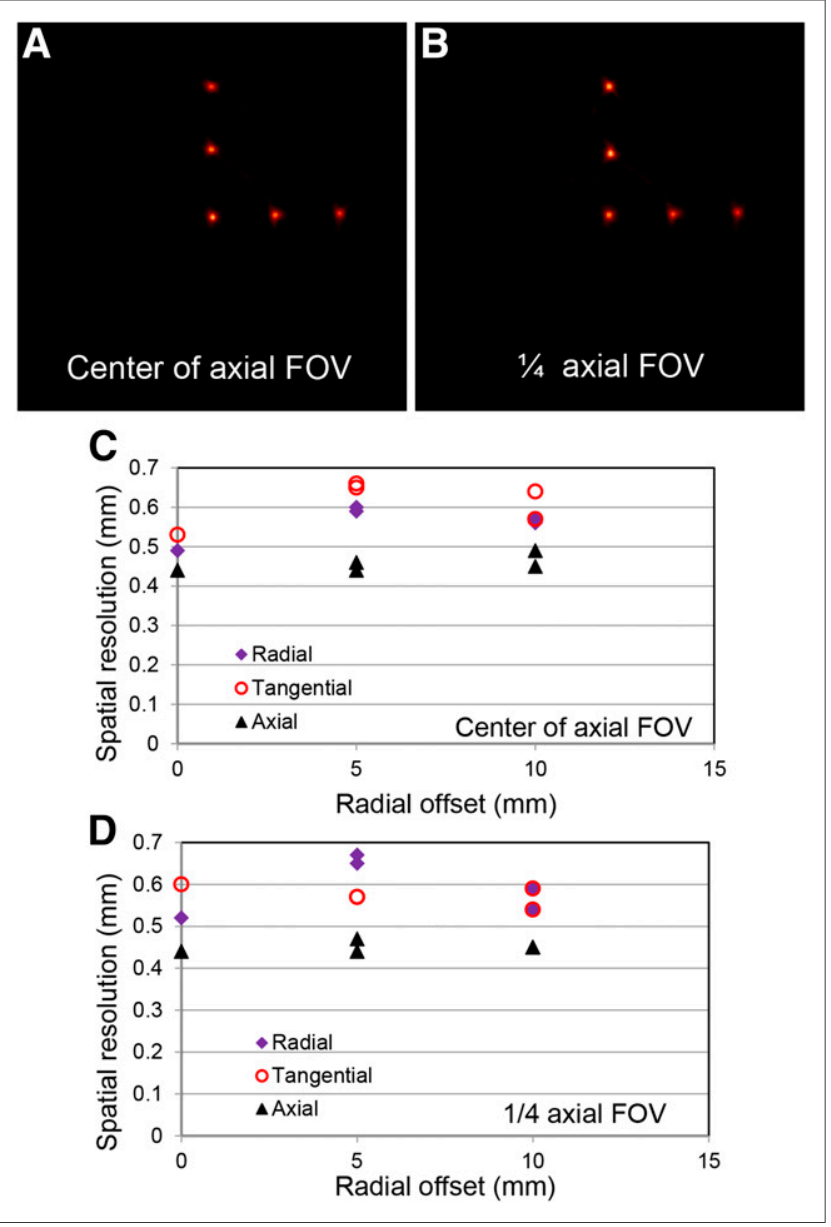

FIGURE 2. Reconstructed PET images from point-source measurements. Radial offsets of point source were 0,5 , and $10 \mathrm{~mm}$. Measurements were made at 2 axial locations, in center of scanner axially (A) and $1.75 \mathrm{~mm}$ away from central slice (B). Graphs showing spatial resolution of scanner at center of axial FOV (C) and in slice at one-quarter axial FOV (1.75 mm from center) (D). Two source positions were measured for radial offsets of 5 and $10 \mathrm{~mm}$ as shown in $\mathrm{A}$ and $\mathrm{B}$; therefore, there are 2 data points at these locations.

look-up table and the photopeak amplitude information to create flood histograms for a specific energy window.

DOI Resolution. The measurements were performed in coincidence mode, using the same source and selectively irradiating at depths of 1.5 , $4,6.5,9$, and $11.5 \mathrm{~mm}$ from the narrow end of the array using electronic collimation. Details on the experimental setup have been published previously (15). The distance from the point source $(0.3 \mathrm{~mm}$ in diameter) to the collimating slab detector $(1.5 \mathrm{~mm}$ thick) was $6 \mathrm{~cm}$, and the distance from the point source to the LSO array was $4 \mathrm{~cm}$. The radiation beam width on the array was estimated from the geometry to be about $1 \mathrm{~mm}$. Histograms of the DOI ratio (ratio of the energy signal of one PSAPD to the sum of the energy signals of both PSAPDs) as a function of irradiation depth were obtained for the entire array and for individual crystals. The DOI resolution was calculated by a gaussian fit of the DOI ratio histograms. The full-width-at-half-maximum (FWHM) DOI resolution was then converted to millimeters by a linear fit of the peak values of the DOI ratio histogram of the entire array to the known depth of irradiation, using the data obtained at depths of 1.5 and $11.5 \mathrm{~mm}$.

\section{Scanner Performance}

Intrinsic Spatial Resolution. The intrinsic spatial resolution of the detectors after installation in the scanner was measured by moving a
0.3 -mm-diameter ${ }^{22} \mathrm{Na}$ point source along the central axis of the scanner in the axial direction with a step size of $0.1 \mathrm{~mm}$. In total, 36 positions in the center of the axial field of view (FOV) were measured. For each position, the coincidence counts measured from each crystal in the 2 middle rows (crystal rows 7 and 8 ) in one detector with the corresponding crystal in the opposite detector were obtained using the look-up tables and a detector-based lower energy threshold of $250 \mathrm{keV}$. The coincidence counts for the 14 crystal pairs in the same row were summed to increase the statistics of the measurements. In total, 16 curves of the coincidence counts versus source position (intrinsic spatial resolution profiles) were obtained ( 8 different detector module pairs $\times 2$ rows of crystals). The FWHM of the profiles was obtained using linear interpolation of the measured data. The measurements presented were not corrected for the $0.3-\mathrm{mm}$ diameter of the point source.

Timing Resolution. A uniform cylinder with a diameter of $30 \mathrm{~mm}$ and a length of $10 \mathrm{~mm}$ (covering the whole FOV of the scanner) was filled with ${ }^{18} \mathrm{~F}$-fluoride and placed in the scanner. The logic OR of the 8 detector timing signals was used as the start signal, and the logic OR of the other 8 timing signals was used as the stop signal, and these signals were fed into a time-to-digital converter. The measured timing spectrum was fit with a gaussian function, and the FWHM timing resolution of the scanner was measured.

Sensitivity. The sensitivity of the scanner was measured by stepping a $0.3-\mathrm{mm}{ }^{22} \mathrm{Na}$ point source along the central axis of the scanner. The sensitivity for 2 different lower energy thresholds, 150 and $250 \mathrm{keV}$, was measured. The $90.3 \% \beta^{+}$decay branching ratio for ${ }^{22} \mathrm{Na}$ was accounted for, but the small degree of attenuation of $\gamma$-rays in the plastic disk containing the point source was not corrected for. The relative sensitivity change of the scanner for different coincidence timing windows was also measured using the same uniform cylinder as for the timing resolution measurement and a lower energy threshold of $150 \mathrm{keV}$. The width of the coincidence window to be used for later studies was based on this measurement.

DOI Calibration. From a GATE (Geant4 Application for Tomographic Emission) simulation (23), the interaction probabilities of $511 \gamma$-rays along the depth of the tapered array used in the scanner were obtained for a point source at the center of the FOV (Supplemental Fig. 3A). The curves of the interaction probabilities versus the DOI ratio (DOI responses) were measured for all 16 detectors by placing a point source at the center of the FOV. Supplemental Figure $3 \mathrm{~B}$ shows the measured DOI response for one detector. The DOI calibration curve of depth versus the DOI ratio can then be obtained by finding the values for the depth and DOI ratio that have the same fraction of counts under the curve. For one detector array, the depth-versus-DOI ratio was actually measured (during the DOI resolution measurement). This represents the gold standard method. Supplemental Figure 3C, a comparison of the calibration curve of that detector obtained by the current method versus the gold standard method, shows excellent agreement between the two and validates this simple approach to DOI calibration. This method was therefore used for DOI calibration of all detectors in the prototype scanner.

Image Reconstruction. For image reconstruction, we apply sinogrambased 3-dimensional (3D) maximum-likelihood expectation maximization (MLEM) using 9 DOI bins. The size of the sinogram dataset was $71 \times 112 \times 9 \times 9 \times 14 \times 14$ elements (radial projection $\times$ angle $\times$ $\mathrm{DOI}_{1} \times \mathrm{DOI}_{2} \times \operatorname{ring}_{1} \times \operatorname{ring}_{2}$ ). The system matrix was precomputed by dividing each depth-encoding crystal into $5 \times 5 \times 11$ subelements to perform the numeric volume integration using Siddon's method (24). The system matrix was compressed with both axial and transaxial symmetries (25) and was saved in sparse matrix format. An image domain point-spread function was also included in the reconstruction. 
The point-spread functions were obtained using a double-gaussian model estimated from reconstructed images of real data from pointsource scans at different locations in the FOV (26). A uniform-cylinder phantom filled with $5.5 \mathrm{MBq}$ of ${ }^{18} \mathrm{~F}$-fluoride was scanned for $4 \mathrm{~h}$ (50 million counts) to obtain normalization data. The cylinder was $30 \mathrm{~mm}$ in diameter and $10 \mathrm{~mm}$ in length, which covered the entire FOV of the scanner. Crystal efficiencies and geometric correction factors were estimated using an iteration-based normalization algorithm (27). Random and scatter correction was not considered in the reconstruction. Each image was reconstructed into a matrix of $149 \times$ $149 \times 29$ voxels with a voxel size of $0.2 \times 0.2 \times 0.25 \mathrm{~mm}$.

Image Spatial Resolution. The spatial resolution was measured following the National Electrical Manufacturers Association NU4 standard for small-animal PET scanners (28). A $0.3-\mathrm{mm}{ }^{22} \mathrm{Na}$ point source was scanned at radial distances of 0,5 , and $10 \mathrm{~mm}$ both at the center of the axial FOV and at an axial offset of $1.75 \mathrm{~mm}$ from the center. Sources were reconstructed (700 iterations) by adding a uniform warm background without noise to the sinogram to ensure that the measured resolution was not artificially improved because of the nonnegativity constraint in the MLEM algorithm. In the reconstructed image, the intensity ratio of the point source to the background was 1:10. The background was subtracted from the reconstructed image. One-dimensional profiles in all 3 directions were obtained through the point-source images, and FWHM image resolution was obtained using a gaussian fit to the profiles. A detectorbased lower energy threshold of $250 \mathrm{keV}$ was used for this and all subsequent imaging studies. The crystal look-up tables of all detectors were created using the flood histograms obtained from the normalization scan.

\section{Phantom Study}

A hot-rod microphantom filled with ${ }^{18} \mathrm{~F}$-fluoride was scanned with both the prototype PET scanner and an Inveon D-PET scanner (Siemens) (2). The rod diameters of the phantom are $0.35,0.40,0.45$, $0.50,0.60$, and $0.75 \mathrm{~mm}$, and the rod-to-rod distance is twice the rod diameter. For the prototype, the scan time was $240 \mathrm{~min}$ and the activity at the start was $4.2 \mathrm{MBq}$. In total, 38 million counts were acquired. For the Inveon D-PET, the scan time was $30 \mathrm{~min}$ and the starting activity was $4.4 \mathrm{MBq}$. In total, 380 million counts were acquired. A longer acquisition time was used for the prototype as it has only one detector ring (and therefore lower sensitivity) and sufficient counts are required to reconstruct at the highest possible resolution. The dataset from the prototype was reconstructed by a 3D MLEM algorithm with 700 iterations. The Inveon D-PET images were reconstructed by 3D ordered-subset expectation maximization (OSEM)/maximum a posteriori (MAP) with the vendor-suggested default reconstruction parameters (2 OSEM iterations and 18 MAP iterations).

\section{In Vivo Mouse Brain Studies}

The prototype has a small axial FOV of $7 \mathrm{~mm}$ and is designed primarily for mouse brain imaging. Activity outside the FOV can contribute to singles, randoms, and scatters. All in vivo mouse brain studies were therefore performed by inserting the mouse body into a tungsten tube to shield activity outside the FOV. This tube was $60 \mathrm{~mm}$ long and had an inner diameter of $25 \mathrm{~mm}$ and an outer diameter of $35 \mathrm{~mm}$. The effectiveness and need for shielding were demonstrated by prior counting-rate-performance data obtained with and without lead shielding (Supplemental Fig. 4). The tungsten tube also served to keep the mouse warm during imaging, as it was warmed by ultra-thin heating sheets to a temperature of $35^{\circ} \mathrm{C}$.

Two types of animal scan were performed under the approval of the University of California-Davis Institutional Animal Care and Use Committee. The mice were anesthetized with $1 \%$ isoflurane during intravenous injection and imaging and were awake during the uptake time.

${ }^{18}$ F-Fluoride Scan. A juvenile 12.6-g mouse was injected with $18 \mathrm{MB}$ of ${ }^{18} \mathrm{~F}$-fluoride and the head positioned for imaging in the prototype. The scan started $120 \mathrm{~min}$ after injection and lasted $60 \mathrm{~min}$. In total, 6.2 million counts were acquired. For comparison, an 11.9-g mouse was injected with $12 \mathrm{MBq}$ of ${ }^{18} \mathrm{~F}$-fluoride and the head positioned in the center of the axial FOV of the Inveon D-PET. The scan started $30 \mathrm{~min}$ after injection and lasted $30 \mathrm{~min}$. In total, 459 million counts were acquired. The image from the prototype was reconstructed by 3D MLEM with 300 iterations, and the image from the Inveon D-PET was reconstructed by 3D OSEM/MAP.

${ }^{18}$ F-FDG Scan. A 19.4-g mouse was injected with $21 \mathrm{MBq}$ of ${ }^{18} \mathrm{~F}-\mathrm{FDG}$ and positioned in the Inveon D-PET for a 15 -min scan starting $30 \mathrm{~min}$ after injection. In total, 498 million counts were acquired. The mouse was then moved to the prototype for a $60-\mathrm{min}$ scan starting $60 \mathrm{~min}$ after injection. In total, 6.3 million counts were acquired. The image from the prototype was reconstructed by 3D MLEM with 60 iterations, and the image from the Inveon D-PET was reconstructed by 3D OSEM/MAP.

\section{RESULTS}

\section{Detector Performance}

Supplemental Figure 5 shows a representative flood histogram from one detector, as well as the energy spectra of a crystal in the center and a crystal in the second row from the edge. The lower energy threshold was set at $250 \mathrm{keV}$ for the events contributing to the flood histogram. All crystals except those in the 2 rows closest to the edge can be clearly resolved. The energy resolution of the center crystals ranges from $20 \%$ to $25 \%$. At the edges, the energy resolution is degraded; the crystals in the 2 rows closest to the edge cannot be clearly resolved, and the photopeak amplitude for the crystals in the row at the very edge is lower than for the other crystals. Supplemental Figure 5 also shows the DOI responses of all crystals measured at 5 depths with electronic collimation (lower energy threshold also $250 \mathrm{keV}$ ). The average DOI resolution over the 5 depths was $1.7 \mathrm{~mm}$. The estimated width of the collimated beam $(\sim 1 \mathrm{~mm})$ was not subtracted from the results. The average DOI resolution obtained from the DOI responses of the individual crystals was $1.5 \mathrm{~mm}$. There were only slight variations among the measured flood histograms and DOI responses of the 16 detectors inside the scanner.

\section{Scanner Performance}

Supplemental Figure 6 shows profiles of the intrinsic spatial resolution measurements for 2 middle rows of crystals (summed over the crystals in that row) from one pair of detectors. In total, 16 profiles were obtained for 8 detector pairs. The average intrinsic spatial resolution of the 16 measured profiles was $0.61 \mathrm{~mm}$, with a maximum of $0.68 \mathrm{~mm}$ and a minimum of $0.54 \mathrm{~mm}$.

The scanner timing resolution was $40 \mathrm{~ns}$. The poor timing resolution is mainly a result of the well-characterized positiondependent time shift of PSAPDs (29-31). Supplemental Figure 7 shows the sensitivity of the scanner for different axial positions. The sensitivity at the center FOV was $1.02 \%$ and $0.68 \%$ for lower energy thresholds of 150 and $250 \mathrm{keV}$, respectively. Supplemental Figure 7 also shows the relative sensitivity measured with a uniformcylinder phantom for different coincidence timing windows. The lower energy threshold for this measurement was $150 \mathrm{keV}$. On 
the basis of this result, a coincidence timing window of $60 \mathrm{~ns}$ was used for all imaging studies.

Figure 2 shows reconstructed images of the point source measured at 5 positions within the scanner, both in the central axial slice and in a slice corresponding to one fourth the axial FOV (1.75 $\mathrm{mm}$ from the center). Figure 2 also shows the measured spatial resolution. The reconstructed axial spatial resolution was about $0.45 \mathrm{~mm}$, which is the best among all 3 directions since the crystal dimensions are not tapered in the axial direction. The radial and tangential spatial resolution was between 0.5 and $0.7 \mathrm{~mm}$ for all positions measured. The spatial resolution at a radial offset of $5 \mathrm{~mm}$ was the worst, probably because of the fairly large gaps between the detector blocks. An average spatial resolution of $0.55 \mathrm{~mm}$ was achieved across a FOV that can accommodate the entire mouse brain.

\section{Phantom and In Vivo Animal Studies}

Figure 3 shows the reconstructed hot-rod microphantom images. For images acquired with the prototype, rods with a diameter of as low as $0.5 \mathrm{~mm}$ can be resolved. Even the largest rods, $0.75 \mathrm{~mm}$, cannot be resolved in the images from the Inveon D-PET because it has a spatial resolution of about $1.5 \mathrm{~mm}$ (2).

Figure 4 shows the ${ }^{18} \mathrm{~F}$-fluoride images. The Inveon D-PET images are 5 contiguous slices with a thickness of $0.80 \mathrm{~mm}$. The slice thickness of the prototype is $0.25 \mathrm{~mm}$, and 1 in every 3 slices is shown. The images from the prototype show much higher spatial resolution, as expected. Profiles were also taken through the skull of the mice (estimated true thickness, $\sim 250 \mu \mathrm{m}$ ). The FWHM of the profiles was $0.6 \mathrm{~mm}$ as measured with the prototype and $1.8 \mathrm{~mm}$ as measured with the Inveon D-PET, indicating that the high resolution measured in phantom studies also can be obtained in vivo.

Figure 5 shows the ${ }^{18} \mathrm{~F}-\mathrm{FDG}$ images. The images obtained with the prototype show much higher spatial resolution and more detailed structure than the images obtained with the Inveon D-PET, but the noise level of this one-ring prototype is clearly higher because of the limited counts that could be collected (6.3 million events for the prototype vs. 498 million events for the Inveon D-PET). The sensitivity of the scanner needs to be significantly increased to take full advantage of the high spatial resolution.

\section{DISCUSSION}

A prototype high-resolution small-animal PET scanner with one detector ring was developed using dual-ended-readout depthencoding tapered detectors. The very small size of the crystal elements

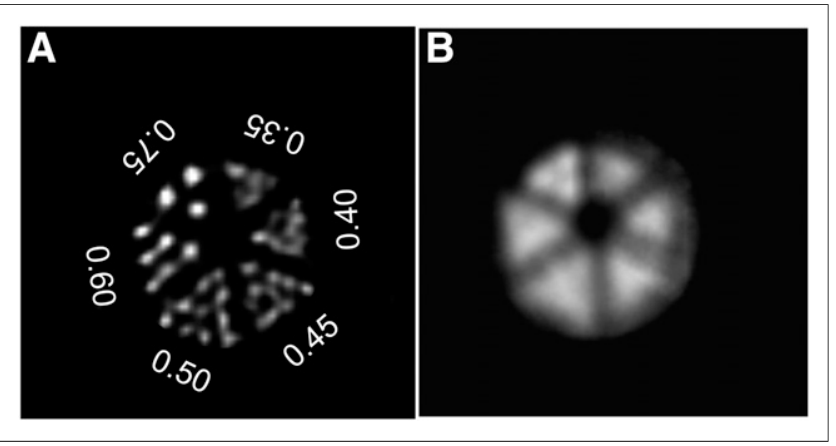

FIGURE 3. Reconstructed PET images of hot-rod phantom obtained using prototype scanner (A) and Inveon D-PET (B). Rod diameters (mm) are indicated in $\mathrm{A}$.

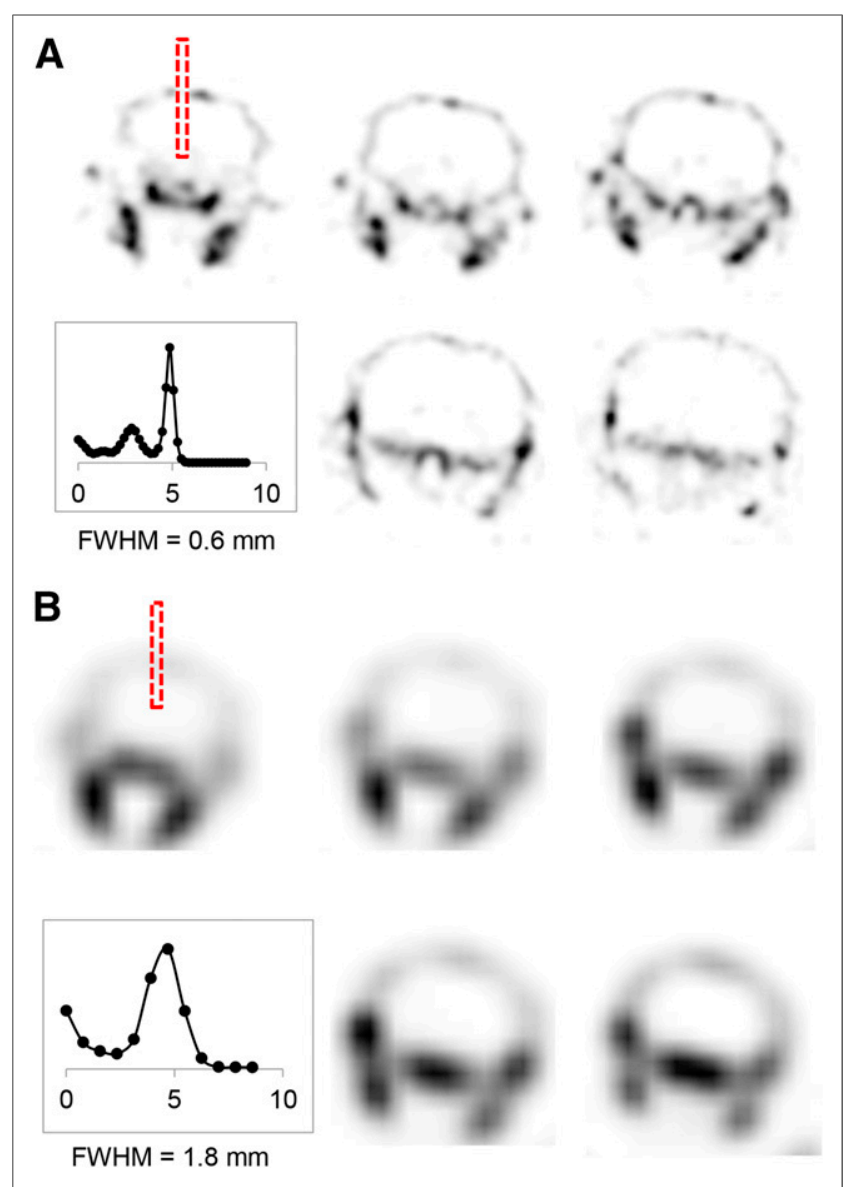

FIGURE 4. Reconstructed PET images of ${ }^{18} \mathrm{~F}$-fluoride uptake in mouse skull obtained using prototype scanner (A) and Inveon D-PET (B). Line profiles through skull are also shown for first slice of each set of images.

( $0.43 \mathrm{~mm}$ at the front end), combined with a 3D MLEM reconstruction algorithm that accurately models system geometry and other physical processes affecting localization of the annihilation photons, led to a reconstructed spatial resolution that averaged $0.55 \mathrm{~mm}$ across a volume that can accommodate the mouse brain. Animal studies confirmed achievement of this resolution in vivo and demonstrated some of the highest-resolution PET scans ever acquired in a living subject, approaching the limits dictated by positron physics and detector interaction physics (7).

Although the spatial resolution is outstanding and our approach provides a platform for future development, this prototype has several significant limitations that must be overcome to produce a robust and high-performance animal PET scanner. First, the fact that there is only one detector ring leads to poor coverage (axial FOV of only $7 \mathrm{~mm}$ ) and low sensitivity. Second, the fact that the electronics cannot allow for crystal-by-crystal-based timing corrections leads to position-dependent timing shifts within the PSAPDs and a poor timing resolution (40 ns). Third, the fact that there are 5-mm gaps between the detector modules leads to reduced solid-angle coverage and thus reduced system sensitivity. To overcome these limitations, we will be focusing on extending the crystal length from $13 \mathrm{~mm}$ to about $20 \mathrm{~mm}$, extending the axial FOV by adding more detector rings, greatly improving the timing resolution by switching from PSAPDs to silicon photomultipliers, and 


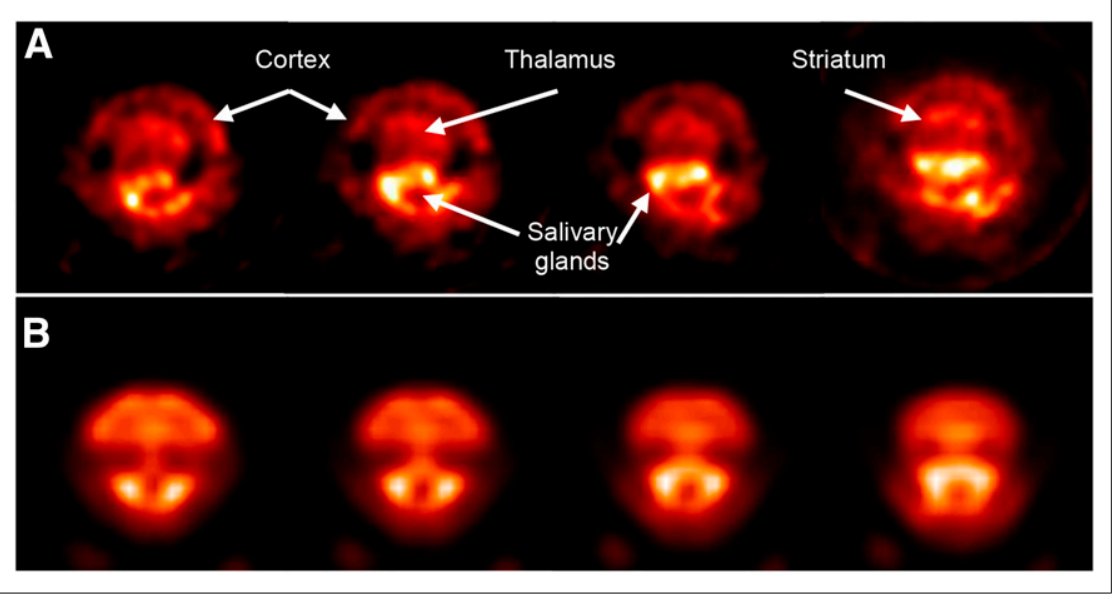

FIGURE 5. ${ }^{18} \mathrm{~F}-\mathrm{FDG}$ PET mouse brain images obtained using prototype scanner (A) and Inveon D-PET (B).

reducing the gaps between detector modules to $1 \mathrm{~mm}$ or less by carefully redesigning the readout electronics (e.g., using the flex readout boards) and using silicon photomultipliers with a through-silicon via and with minimal dead space.

\section{CONCLUSION}

The prototype meets its design objective of achieving a spatial resolution approaching the small-bore PET scanner limits set by positron physics and detector interaction physics. Future development based on this approach should be able to combine the high spatial resolution already demonstrated with better sensitivity and better counting rate performance to produce a high-performance system appropriate for a broad array of applications in the biomedical sciences.

\section{DISCLOSURE}

The costs of publication of this article were defrayed in part by the payment of page charges. Therefore, and solely to indicate this fact, this article is hereby marked "advertisement" in accordance with 18 USC section 1734 . This work was funded by NIH grant R01 EB006109. No other potential conflict of interest relevant to this article was reported.

\section{REFERENCES}

1. Goertzen AL, Bao QN, Bergeron M, et al. NEMA NU 4-2008 comparison of preclinical PET imaging systems. J Nucl Med. 2012;53:1300-1309.

2. Bao Q, Newport D, Chen M, Stout DB, Chatziioannou AF. Performance evaluation of the Inveon dedicated PET preclinical tomograph based on the NEMA NU-4 standards. J Nucl Med. 2009;50:401-408.

3. Szanda I, Mackewn J, Patay G, et al. National Electrical Manufacturers Association NU-4 performance evaluation of the PET component of the NanoPET/CT preclinical PET/CT scanner. J Nucl Med. 2011;52:1741-1747.

4. Wong $\mathrm{WH}, \mathrm{Li} \mathrm{HD}$, Baghaei $\mathrm{H}$, et al. Engineering and performance (NEMA and animal) of a lower-cost higher-resolution animal PET/CT scanner using photomultiplierquadrant-sharing detectors. J Nucl Med. 2012;53:1786-1793.

5. Larobina M, Brunetti A, Salvatore M. Small animal PET: a review of commercially available imaging systems. Curr Med Imaging Rev. 2006;2:187-192.

6. Lewellen TK. Recent developments in PET detector technology. Phys Med Biol. 2008;53:R287-R317.
7. Stickel JR, Cherry SR. High-resolution PET detector design: modelling components of intrinsic spatial resolution. Phys Med Biol. 2005;50:179-195.

8. Eriksson L, Wienhard K, Eriksson M, et al. The ECAT HRRT: NEMA NEC evaluation of the HRRT system, the new high-resolution research tomograph. IEEE Trans Nucl Sci. 2002;49:2085-2088.

9. Cherry SR. The 2006 Henry N. Wagner lecture: of mice and men (and positrons) - advances in PET imaging technology. J Nucl Med. 2006;47:17351745.

10. Tai YC, Laforest R. Instrumentation aspects of animal PET. Annu Rev Biomed Eng. 2005;7:255-285.

11. Peng BH, Levin CS. Recent developments in PET instrumentation. Curr Pharm Biotechnol. 2010;11:555-571.

12. España S, Marcinkowski R, Keereman V, Vandenberghe S, Van Holen R. DigiPET: submillimeter spatial resolution small-animal PET imaging using thin monolithic scintillators. Phys Med Biol. 2014;59:3405-3420.

13. Yamamoto $\mathrm{S}$, Watabe $\mathrm{H}$, Kanai $\mathrm{Y}$, Watabe $\mathrm{T}$, Kato K, Hatazawa J. Development of an ultrahigh resolution Si-PM based PET system for small animals. Phys Med Biol. 2013;58:7875-7888.

14. Moses WW. Fundamental limits of spatial resolution in PET. Nucl Instrum Methods Phys Res A. 2011;648(suppl):S236-S240.

15. Yang Y, Dokhale PA, Silverman RW, et al. Depth of interaction resolution measurements for a high resolution PET detector using position sensitive avalanche photodiodes. Phys Med Biol. 2006;51:2131-2142.

16. Yang Y, St James S, Wu YB, et al. Tapered LSO arrays for small animal PET. Phys Med Biol. 2011;56:139-153.

17. St James S, Yang YF, Wu YB, et al. Experimental characterization and system simulations of depth of interaction PET detectors using $0.5 \mathrm{~mm}$ and $0.7 \mathrm{~mm}$ LSO arrays. Phys Med Biol. 2009;54:4605-4619.

18. Shah KS, Grazioso R, Farrell R, et al. Position sensitive APDs for small animal PET imaging. IEEE Trans Nucl Sci. 2004;51:91-95.

19. Yang Y, Wu YB, Farrell R, Dokhale PA, Shah KS, Cherry SR. Signal and noise properties of position-sensitive avalanche photodiodes. Phys Med Biol. 2011;56: 6327-6336.

20. Wu Y, Catana C, Cherry SR. A multiplexer design for position-sensitive avalanche photodiode detectors in a PET scanner. IEEE Trans Nucl Sci. 2008;55: 463-468.

21. Judenhofer MS, Pichler BJ, Cherry SR. Evaluation of high performance data acquisition boards for simultaneous sampling of fast signals from PET detectors. Phys Med Biol. 2005;50:29-44.

22. Ren S, Yang YF, Cherry SR. Effects of reflector and crystal surface on the performance of a depth-encoding PET detector with dual-ended readout. Med Phys. 2014;41:072503.

23. Jan S, Santin G, Strul D, et al. GATE: a simulation toolkit for PET and SPECT. Phys Med Biol. 2004;49:4543-4561.

24. Siddon RL. Fast calculation of the EXACT radiological path for a 3-dimensional CT array. Med Phys. 1985;12:252-255.

25. Johnson CA, Yan YC, Carson RE, Martino RL, Daubewitherspoon ME. A system for the 3D reconstruction of retracted-septa PET data using the EM algorithm. IEEE Trans Nucl Sci. 1995;42:1223-1227.

26. Reader AJ, Julyan PJ, Williams H, Hastings DL, Zweit J. EM algorithm system modeling by image-space techniques for PET reconstruction. IEEE Trans Nucl Sci. 2003;50:1392-1397.

27. Bai B, Li Q, Holdsworth $\mathrm{CH}$, et al. Model-based normalization for iterative 3D PET image reconstruction. Phys Med Biol. 2002;47:2773-2784.

28. NEMA Standards Publication NU 4-2008: Performance Measurements of Small Animal Positron Emission Tomography. Rosslyn, VA: National Electrical Manufacturers Association; 2008.

29. Catana C, Wu YB, Judenhofer MS, Qi JY, Pichler BJ, Cherry SR. Simultaneous acquisition of multislice PET and MR images: initial results with a MRcompatible PET scanner. J Nucl Med. 2006;47:1968-1976.

30. Yang Y, Wu YB, Qi J, et al. A prototype PET scanner with DOI-encoding detectors. J Nucl Med. 2008;49:1132-1140.

31. Wu Y, Ng TSC, Yang YF, Shah K, Farrell R, Cherry SR. A study of the timing properties of position-sensitive avalanche photodiodes. Phys Med Biol. 2009;54: $5155-5172$. 